\title{
Contrast-enhanced ultrasound of focal liver masses
}

\author{
Stephanie R. Wilson ${ }^{1}$, Christina Merrill² \\ 'Department of Radiology, University of Calgary, Calgary T2N 2T9, Canada. \\ ${ }^{2}$ Department of Radiology, Foothills Medical Centre, Calgary T2N 2T9, Canada.
}

Correspondence to: Prof. Stephanie R. Wilson, Department of Radiology, University of Calgary, 140329 Street NW, Calgary T2N 2T9, Canada. E-mail: stephanie.wilson@ahs.ca

How to cite this article: Wilson SR, Merrill C. Contrast-enhanced ultrasound of focal liver masses. Hepatoma Res 2020;6:57. http://dx.doi.org/10.20517/2394-5079.2020.48

Received: 4 May 2020 First Decision: 3 Jun 2020 Revised: 22 Jun 2020 Accepted: 6 Jul 2020 Published: 1 Sep 2020

Academic Editor: Yuko Kono Copy Editor: Cai-Hong Wang Production Editor: Jing Yu

\begin{abstract}
Non-invasive imaging is the current method of choice for the characterization of frequently discovered focal liver disease. Although historically, contrast-enhanced computed tomography (CT) and magnetic resonance (MR) scans have been selected for this purpose, contrast-enhanced ultrasound (CEUS) now offers a less expensive and safer method to acquire the same information. Performed with the intravenous injection of a microbubble contrast agent, CEUS provides some unique advantages that make it a valuable addition to an imaging toolbox. CEUS is performed in dynamic real-time, providing superior temporal resolution compared to other modalities and allowing detection of enhancement regardless of its timing or duration. CEUS is performed with a purely intravascular contrast agent, providing accurate depiction of the presence of microbubbles in the circulation in all phases of imaging. This compares with CT and MR contrast agents, which have a well-recognized interstitial phase. Resulting discordant imaging may occur especially in the portal venous phase, when CT and MR may show pseudoenhancement from interstitial contrast, while CEUS will accurately show washout in malignant tumors. Lastly, the contrast specific software used to perform CEUS has an excellent subtraction technique, which produces a contrast only image with high sensitivity to enhancement in thin septations and small nodules. CEUS makes a positive contribution to liver mass characterization in any situation.
\end{abstract}

Keywords: Contrast-enhanced ultrasound, liver, cancer, diagnosis

\section{INTRODUCTION}

Focal liver masses are a frequent occurrence and reflect a wide spectrum of histology ranging from benign and totally insignificant tumors to life-threatening malignancies. Incidental discovery on imaging, 
performed for an unrelated reason, is common, especially for benign tumors. Alternately, tumors may be found as a result of a directed search in those with symptoms, with primary malignancy elsewhere, or in those identified to be at risk for hepatocellular carcinoma (HCC).

Regardless of the nature or method of discovery of a focal liver mass, contrast-enhanced imaging is now a mainstay for its noninvasive characterization. Originally, computed tomography (CT) and magnetic resonance (MR) scans comprised the recommended modalities for this purpose. However, since the introduction of microbubble contrast agents for ultrasound (US) two decades ago, CEUS has become an additional lower cost and safer option with unique benefits ${ }^{[1]}$.

The late approval of a contrast agent for CEUS in the United States in 2016 makes this an expanding but still introductory modality in North America today. However in other parts of the world, such as Europe, the role of CEUS has been well established by international and national guidelines ${ }^{[2-4]}$. Here, we describe the CEUS technique, emphasizing its unique benefits for characterization of focal liver disease.

\section{Ultrasound contrast agents: what are they and how do they work?}

Ultrasound contrast agents are comprised of tiny encapsulated bubbles of a perfluoropropane gas within a variable, usually lipid, shell. They are on the same order of size as a red blood cell, and their distribution in the circulation is sparse. They are purely intravascular as their size does not allow them to pass into the interstitial tissues. After their venous injection, they are quickly exhaled by the lungs. They are very safe in clinical practise for adults and for children, they have no nephrotoxicity, and their imaging requires no ionizing radiation ${ }^{[5-7]}$.

\section{Specialized imaging techniques}

The successful imaging of microbubble contrast agents is facilitated by their oscillation when they are viewed in a low-power US field. To detect the signal from the oscillating microbubbles in the circulation, CEUS is performed with contrast specific software ${ }^{[8]}$. This generally includes pulse inversion imaging, whereby two pulses are sent down each scan line with subtraction of the returning echoes ${ }^{[9]}$. The background tissue, a linear reflector, sends back a wave which is a reflection of the insonating wave. Their subtraction, therefore, equals zero, totally removing the background signal. The result is a relatively black image at the beginning of an imaging sequence for CEUS. The returning echoes from the oscillating microbubble contrast agents, by comparison, will produce additive signals so that there is great augmentation of the Doppler signal from blood. The result of these sequences is the creation of an incredibly sensitive microbubble only image, with removal of the signals from the background tissues. The intensity of the signal on the image occurs in direct proportion to the volume of microbubbles within the region of view. Today, all high-end US systems offer a contrast specific software package with low mechanical index (MI) imaging and also a bubble tracking technique. This bubble tracking may involve a transient brief high MI insonation which destroys bubbles within the field of view, following which the refilling of the vessels may be tracked, improving their visualization.

Contrast-enhanced imaging diagnosis of all liver masses on all modalities focuses on their enhancement following contrast injection. Although CEUS follows the very same principles as CT and MR scan for diagnoses, with similar result, CEUS shows, in addition, unique capabilities that give it a special place for this application ${ }^{[10]}$.

\section{Unique capabilities of CEUS}

Dynamic real-time imaging

Microbubble contrast agents allow US imaging of organ and lesion perfusion in real time. In comparison to CT or MR which creates single snapshots in time, CEUS shows all images as dynamic real-time studies. 
Therefore, CEUS has superior temporal resolution compared to CT and MR scan, and it has, consequently, the ability to show enhancement changes regardless of their timing or intensity.

\section{Subtraction technique}

CEUS uses a subtraction technique which effectively removes all of the background echogenicity such that all imaging reflects microbubbles only. This microbubble only image provides exceptional sensitivity for CEUS to show enhancement even in very small nodules and thin septations.

Purely intravascular contrast agent

CEUS is performed with purely intravascular contrast agents which will therefore always reflect the presence of contrast agent in a tumor or the liver vasculature. Contrast agents for CT and MR, by comparison, have a well-recognized interstitial phase whereby contrast may leak out of the vasculature into the interstitium of a tumor. The resultant discordant imaging between CEUS and CT/MRI scan makes a very positive contribution to the diagnosis of focal liver masses ${ }^{[11]}$.

These unique features of CEUS imaging require that CEUS have its own algorithms.

\section{CEUS IMAGING OF FOCAL LIVER MASSES}

CEUS of a focal liver mass is based on the enhancement changes that occur over time in a nodule, which is preidentified on greyscale US and maintained within the field of view for the entire scan. These changes are described for the nodule relative to the adjacent liver parenchyma. Initial imaging of the pre-identified nodule should be performed continuously, from contrast injection until peak arterial phase enhancement. Subsequent imaging is intermittent (5-10 s every 30-60 s) to detect any washout and assess its degree, until the end of useful enhancement, generally around 5 to 6 min.

This described technique, with only a brief initial acquisition of multiframe continuous images, audio video interleave (avi), will minimize microbubble destruction. This improves the ability to detect late washout and assess its degree.

Additional valuable scanning techniques include sweeping the entire liver in the portal/late phase with suspended inspiration to identify additional areas of abnormal enhancement, especially washout. Furthermore, injections can be repeated as needed including "on top" of a prior injection to assess vascularity of an identified area of washout.

\section{ALGORITHMS FOR DIAGNOSIS OF FOCAL LIVER MASSES ON CEUS}

Experience with the use of microbubble contrast agents for liver mass characterization has led to the recognition that multiple constant features lead to reliable differentiation of benign and malignant masses and also to their specific accurate diagnoses. Resultant algorithms for diagnosis of focal liver tumors on contrast imaging are all based on interpretation of enhancement features of an identified nodule relative to the adjacent liver from the injection of the contrast agent to the end of useful enhancement, generally around 5 or 6 min following injection ${ }^{[12]}$. Time zero corresponds to the beginning of the saline flush following the contrast injection ${ }^{[13]}$. On CEUS, all timing is given in seconds and minutes rather than in phases. Nonetheless, we include both specific timing and phases for completeness and modality comparisons.

Arterial phase (AP) (from 10 to $20-30$ to $45 \mathrm{~s}$ ) ${ }^{[13]}$ observations include:

Enhancement (yes or no)

(1) Intensity (hyper, iso, hypo, or nonenhancing)

(2) Pattern of AP hyperenhancement (APHE) (Diffuse, nodule-in-nodule, rim, peripheral discontinuous globular, or stellate) 
(3) Direction of vascular filling (centrifugal or centripetal)

Portal venous phase (PVP) (45 s - $2 \mathrm{~min}$ ) and Late phase (LP) (from 2 min to 5 or $6 \mathrm{~min}$ ) observations include:

Washout (yes or no)

(1) Timing (rapid, $<1 \mathrm{~min}$; or late, $>1 \mathrm{~min}$ )

(2) Intensity (weak, with some bubbles remaining in a nodule which is less enhanced than the liver; or marked, whereby the nodule appears black or punched out by $2 \mathrm{~min}$ )

\section{Importance of the clinical scenario}

Additionally, for all contrast-enhanced liver imaging, interpretations must be made with knowledge of demographic and clinical information. Age and sex are helpful as are relevant health events with the greatest importance given to a history of cancer and also risk factors for development of HCC. The clinical indication for a scan, whether it be for surveillance for metastatic tumor or HCC or related to symptoms may also be directive. The algorithmic interpretation of CEUS should always be guided by what is a reasonable answer for the patient under study.

\section{FUNDAMENTALS FOR INTERPRETATION OF CEUS OF FOCAL LIVER DISEASE}

\section{Principle 1. The intensity in a CEUS image reflects the number of microbubbles in the field of view}

As these are exclusively in blood vessels, echo-enhancement is therefore indicative of the volume of blood and its change with time indicative of the rate of perfusion in the region of interest. All interpretations compare nodule enhancement levels with the adjacent and enhancing liver parenchyma.

\section{Principle 2. Determination of malignancy: importance of washout}

Most malignant masses are identified by washout of the mass in the portal venous or late phase ${ }^{[14,15]}$. Washout refers to the decline in the enhancement of a mass relative to that of the adjacent liver parenchyma, following initial AP enhancement. Therefore, if washout is present, malignancy should be considered likely [Figures 1 and 2]. Conversely, if washout is not present and if the mass shows sustained enhancement, there is a high likelihood that it is benign ${ }^{[16]}$. These rules are guidelines and there are, of course, exceptions. These exceptions to this important rule are very important, since occasionally, benign tumors, focal nodular hyperplasia (FNH), hemangioma, and most often adenoma, may show washout, and some malignant tumors, especially precursor nodules for HCC and well-differentiated HCC, may show no washout. Nonetheless, the fundamental rule is invaluable.

\section{Principle 3. Differentiation of malignancy: importance of timing and intensity of washout}

Although CT and MR scan also determine the presence of washout, CEUS additionally evaluates the intensity and timing of washout, further differentiating hepatocellular from nonhepatocellular malignancy ${ }^{[17]}$. HCC tends to show late (later than $1 \mathrm{~min}$ ) and weak washout (so that some bubbles remain in the washout zone) [Figure 1, Video 1], whereas all nonhepatocellular malignancies, including intrahepatic cholangiocarcinoma (ICC), lymphoma and metastasis are characterized by rapid (earlier than $1 \mathrm{~min}$ ) and marked washout, so that all bubbles are absent from the nodule, making it appear as a black, punched out hole [Figure 2, Video 2] $]^{[1,19]}$. This rapid washout may even occur so early as to be within the traditionally defined AP, prior to $30 \mathrm{~s}$.

\section{Principle 4. Detection of malignancy}

A natural consequence of the two previous principles is that the portal venous phase is the optimal time to detect metastases, when their conspicuity will be increased relative to the enhanced background parenchyma ${ }^{[20]}$. This increased conspicuity makes sweeping of the entire liver at this time the ideal 

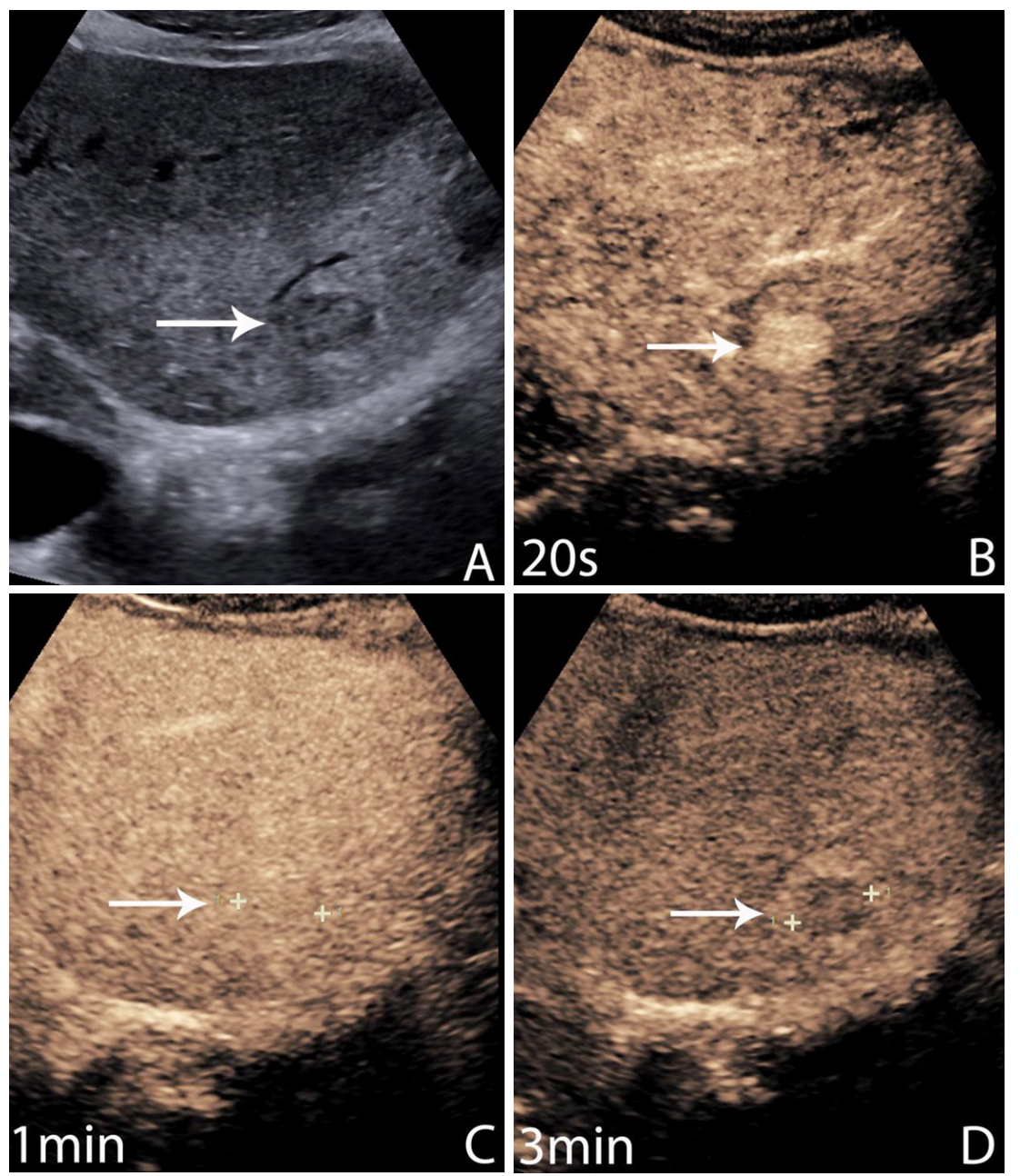

Figure 1. Hepatocellular carcinoma (HCC). 64-year-old male with a history of cirrhosis related to alcohol and fatty liver. Mass found on regular US surveillance. A greyscale image shows a small hypoechoic liver nodule in a very heterogeneous liver (A); at the peak of arterial phase (AP) enhancement, $20 \mathrm{~s}$, the mass is brightly enhanced (B); at one minute, there is isoenhancement. The nodule is now invisible but marked by the arrow (C); there is late and weak washout at 3 min. Weak washout shows less enhancement than the immediately adjacent liver parenchyma but retains bubble signals within the nodule as here. AP hyperenhancement and late weak washout comprise the diagnostic features of HCC (D)

technique and timing for improved detection of metastatic liver masses on CEUS. Although CEUS is not generally involved in the protocolled search for metastases which are developed for follow-up of common tumors of the colon, lung and breast, in particular, it has been shown that CEUS is comparable to CT scan in tumor detection and exceeds CT in some instances ${ }^{[21]}$. Additionally, CEUS plays an important role in the oncology patient, resolving many indeterminate CT and MR scans and generally problem-solving in this population. In our department, CEUS is invaluable to resolve the low-attenuation small indeterminate masses shown regularly on portal venous phase CT scans, easily differentiating small cysts from solid tumors with CEUS features of metastatic disease ${ }^{[22]}$.

\section{Principle 5. Diagnosis of benign tumors}

Benign tumors commonly encountered on CEUS examinations include hemangioma, focal nodular hyperplasia and, much less often, hepatic adenoma. It is recognized that CEUS has a unique capability for their diagnosis in that all may demonstrate highly suggestive enhancement patterns in the $\mathrm{AP}^{[23]}$. Optimally shown by the real-time dynamic scanning afforded by CEUS. Recognizing the significance of washout as an indicator of malignancy, it follows that sustained enhancement, such that the lesion will 

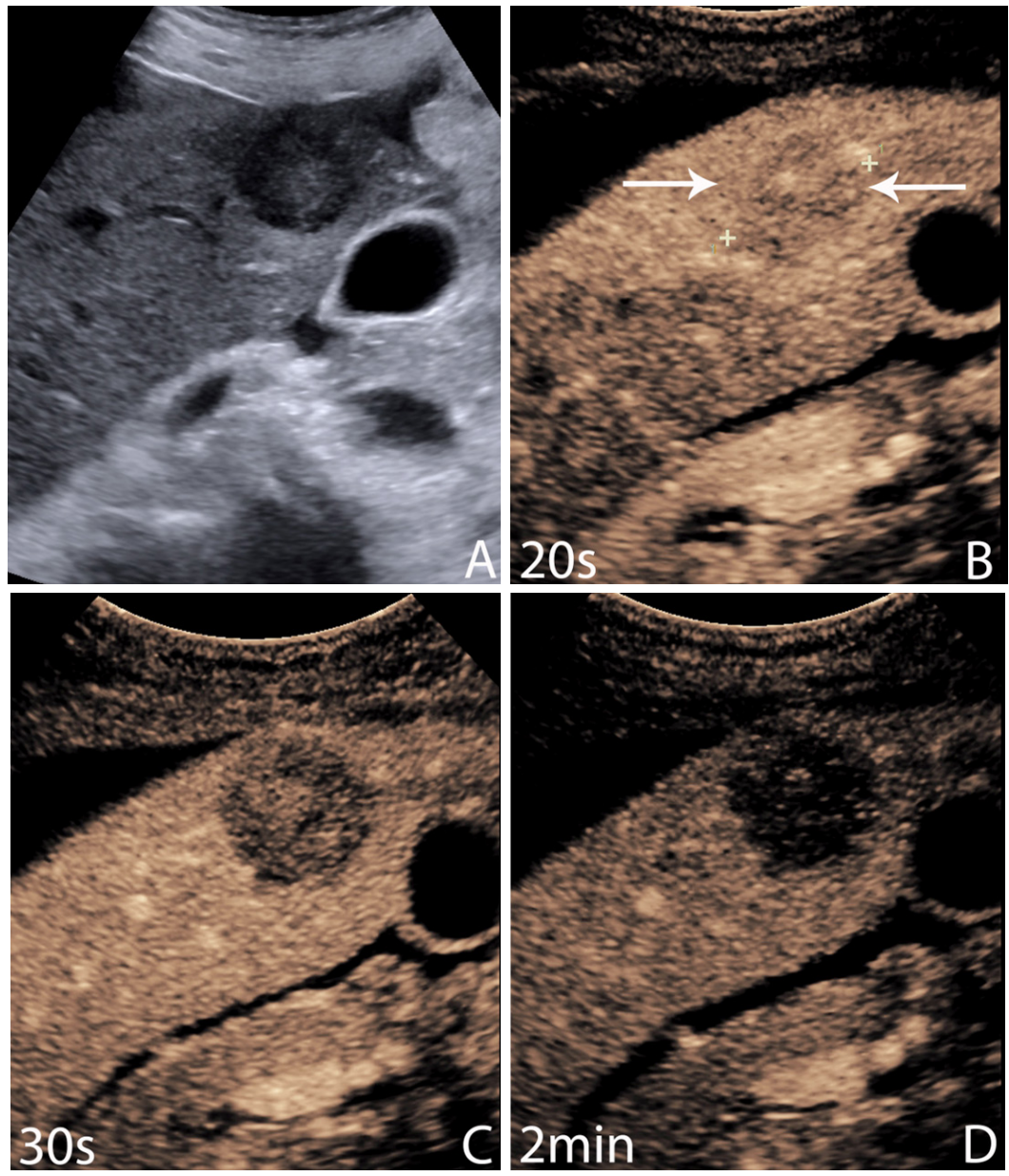

Figure 2. Metastasis. 70-year-old female with abdominal ultrasound performed for right upper quadrant pain, query gallstones. Greyscale ultrasound shows a focal hypoechoic mass in the right lobe of the liver (A); at $20 \mathrm{~s}$, the mass is hypoenhanced, appearing very slightly less bright than the liver (B); at $30 \mathrm{~s}$, there is already washout of the mass, so that it is now much less enhanced than the adjacent liver. It is also less enhanced than it was at $20 \mathrm{~s} \mathrm{(C);} \mathrm{at} 2 \mathrm{~min}$, the mass appears as a black hole due to marked washout. This rapid transition from enhancement to washout is typical of metastatic disease. Biopsy showed adenocarcinoma, of unknown origin (D)

remain as enhanced as the adjacent liver to 4 or even 5 min following injection, is highly associated with benign outcome. Hemangiomas are characterized by peripheral nodular enhancement with centripetal progression of this enhancement over time [Figure 3, Video 3]. If these enhancement patterns are rapidly changing, they may not be appreciated on CT and MR scans, both of which obtain snapshots in time. This is especially important for the recognition of rapid, or "flash", filling hemangiomas on CEUS which may show only as a zone of APHE on CT/MRI scan.

FNH is a highly vascular tumor and is characterized by stellate vessel morphology with filling of the lesion from its center to the periphery ${ }^{[24]}$ [Figure 4, Video 4].

Hepatic adenoma, a fairly rare liver tumor, is an exception to these general rules and may show diffuse enhancement or a more specific filling from the periphery to the center of the lesion [Figure 5, Video 5]. Additionally, adenoma is shown to have the possibility for washout, occurring in up to $50 \%$ of cases ${ }^{[25]}$.

The above described benign tumors, FNH and adenoma, are most often detected incidentally, often on imaging performed for a totally unrelated reason. Adenoma has the additional association of long history of use of oral contraceptives. Both of these tumors have a propensity to occur in asymptomatic young 


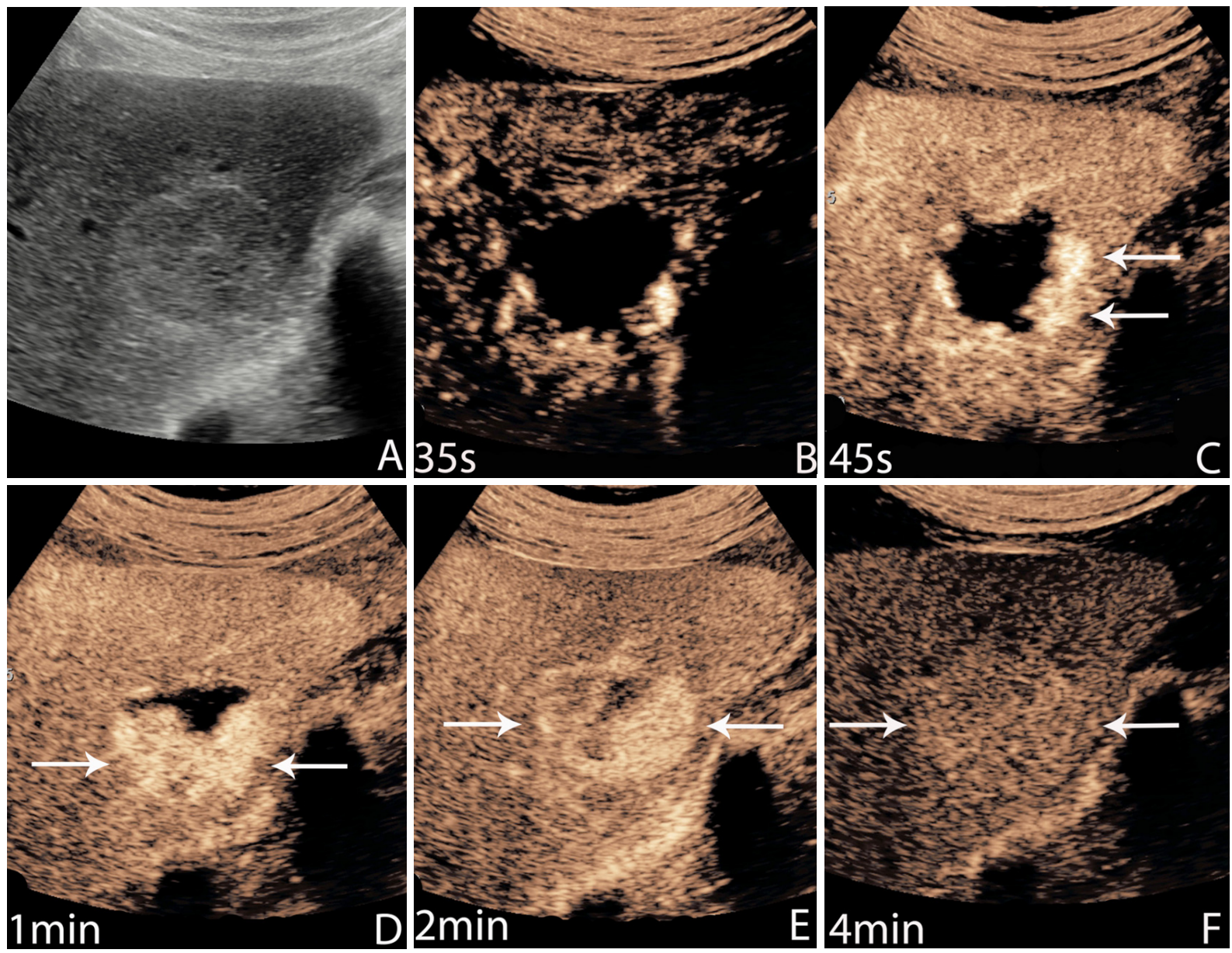

Figure 3. Hemangioma. 39-year-old asymptomatic female with an incidentally discovered liver mass during an US examination for unrelated reasons. A greyscale image shows a focal slightly hypoechoic mass with a thin echogenic rim. It is suggestive of an atypical hemangioma (A); an early AP image, at $35 \mathrm{~s}$, shows a rim of peripheral enhancement with tiny peripheral puddles (B); there is centripetal progression by $45 \mathrm{~s}$ with no linear vascularity $(C)$; by $1 \mathrm{~min}$, there is further centripetal progression with eccentric growth of the peripheral puddles of enhancement (D); by $2 \mathrm{~min}$, there is virtually complete fill in with non-uniform enhancement (E); there is sustained enhancement to $4 \mathrm{~min}$. Peripheral nodular enhancement with sustained enhancement is the classic description of an insignificant hemangioma. Hemangiomas may fill very rapidly, within seconds, or very slowly as here, over several minutes (F)

females, and CEUS ranks with MRI performed with a liver specific agent with a dual elimination pathway for accurate differentiation of the two.

\section{Principle 6. Precise diagnosis of malignant tumors}

Unlike benign lesions, malignant tumors are not generally characterized as reliably by their AP enhancement pattern, which may be highly variable, but instead by the identification of washout in the portal venous and late phases ${ }^{[2]}$. Nonetheless, HCC most often shows a globular pattern of APHE [Figure 1, Video 1]. Nonhepatocellular malignancies may show a similar pattern but also frequently show highly suggestive rim enhancement in the $\mathrm{AP}^{[27]}$.

\section{Principle 7. Discordance of imaging between CEUS and CT/MRI scan}

Discordance of imaging between CEUS and CT/MRI scan in the portal venous phase is often related to differences in the mechanism of action of the contrast agents for each modality ${ }^{[11]}$. Microbubble contrast agents for ultrasound are purely intravascular agents and they do not have any interstitial phase. This is unlike contrast agents for CT and MR scanning where there is a well-recognized interstitial phase, 

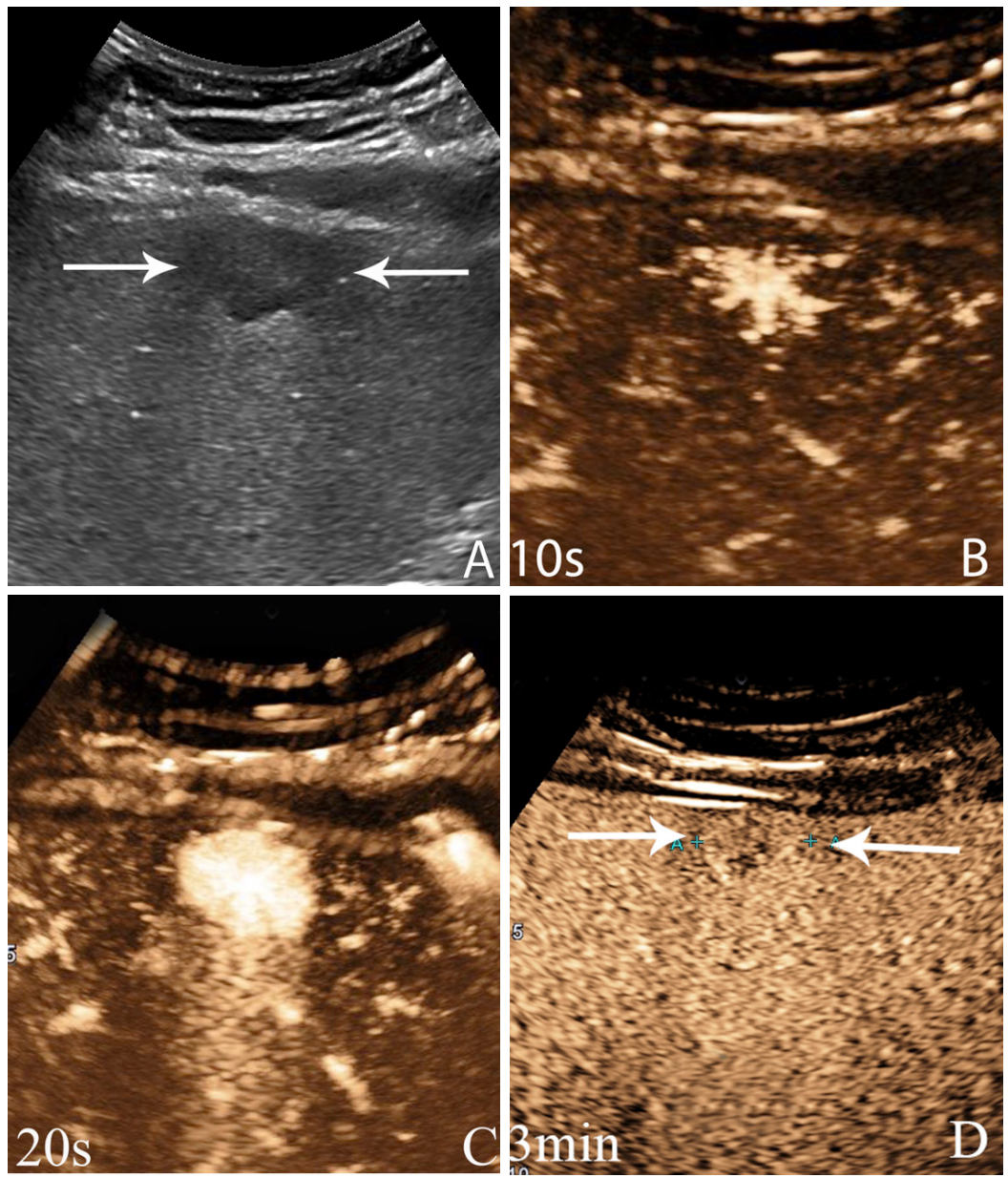

Figure 4. Focal nodular hyperplasia. 25-year-old woman with an incidentally discovered liver mass found during ultrasound performed for examination of her bowel. An incidentally discovered superficial focal hypoechoic liver mass (A); an early arterial phase (AP) image shows a stellate pattern of linear vessels in the center of the mass (B); at the peak of AP enhancement, the mass is brightly and uniformly enhanced (C); at $3 \mathrm{~min}$, the mass continues to show sustained enhancement greater than the adjacent liver (D)

especially evident in malignant tumors with permeable vascular endothelium and a fibrous stroma, such as ICC. Here, the contrast agent leaks into the interstitium, creating a type of pseudoenhancement in the late phase. Thereby, CEUS will show APHE and rapid washout for ICC whereas CT and MR scan may show instead sustained enhancement. This sustained enhancement is in fact pseudoenhancement related to the presence of interstitial contrast agent. This produces a valuable discordance with MR scan of these tumors where washout is appropriately recognized in association with malignant tumors on CEUS only ${ }^{[28]}$. In our own unpublished data, this is most important for diagnosis of cholangiocarcinoma in any liver, either cirrhotic or normal.

\section{ACCURACY OF CEUS}

CEUS shows comparable performance with CT and MR for liver mass characterization and for the prediction of malignancy ${ }^{[26,29]}$. In a prospective multicenter trial with 1,349 patients, there was no statistically significant difference between CEUS and spiral CT in liver tumor differentiation (malignant or benign) and tumor specification ${ }^{[30]}$. In a meta-analysis of 21 studies, CEUS showed a sensitivity of $88 \%$ (95\%CI: $87 \%-90 \%)$ and specificity of $81 \%$ (95\%CI: 79\%-84\%), and diagnostic odds ratio (DOR) was 38.62 (95\%CI: $13.64 \%-109.35 \%$ ), while all parameters were similar with no statistical difference from CECT or CEMRI ${ }^{[31]}$. Furthermore, although of secondary importance to characterization, CEUS is shown to 

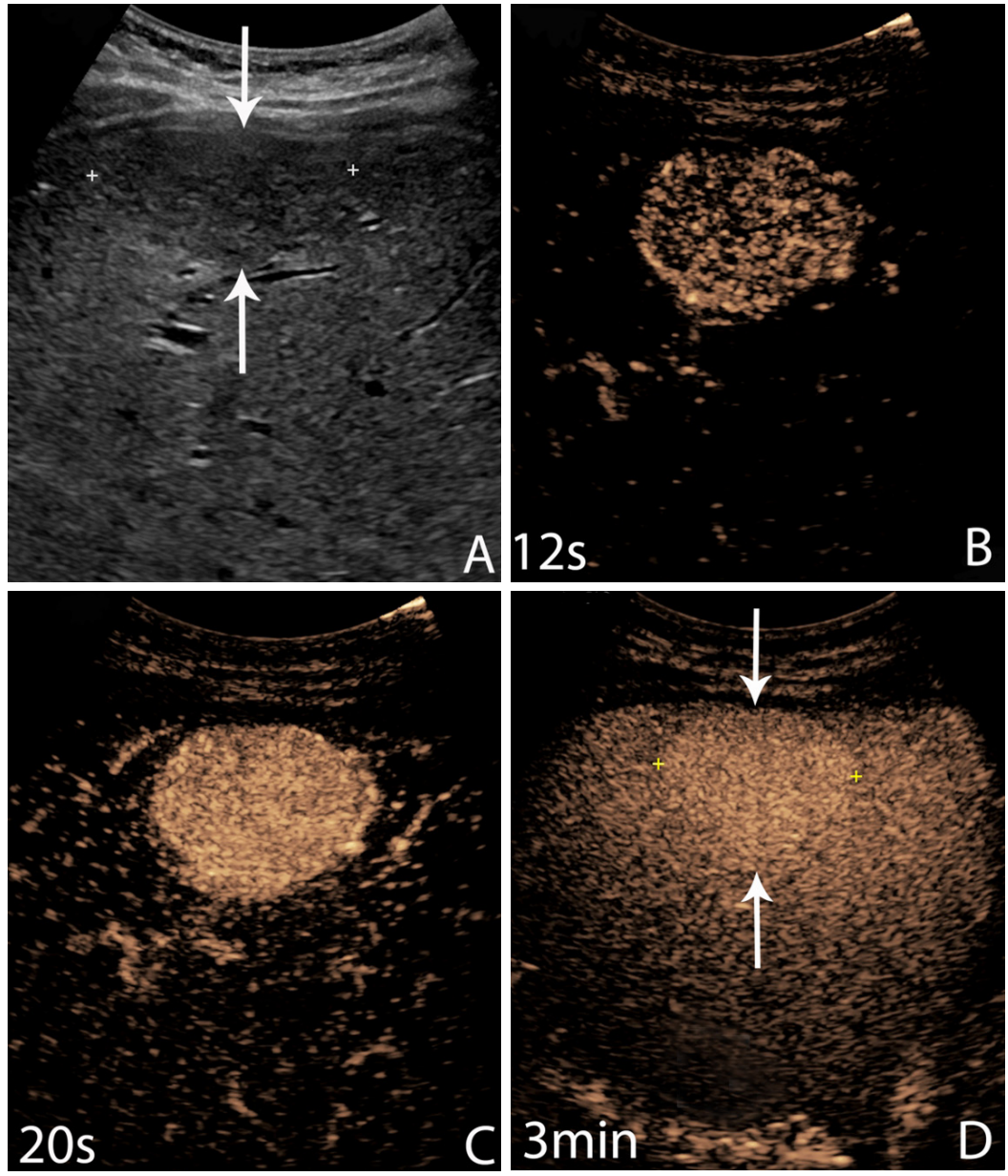

Figure 5. Adenoma. 34-year-old woman with a long continuous history of oral contraceptive use. a greyscale image shows a superficial focal hypoechoic mass (arrows) in a fatty liver (A); an early arterial phase (AP) image at $12 \mathrm{~s}$ shows peripheral irregular vessels (B); at the peak of AP enhancement, $20 \mathrm{~s}$, the mass is uniformly hyperenhanced (C); at $3 \mathrm{~min}$, the mass shows sustained enhancement such that it is still slightly brighter than the adjacent enhanced liver parenchyma (D)

have equivalent, and in some cases superior, performance to CECT and CEMR scan in the detection of metastatic disease.

In conclusion, CEUS provides for the determination of malignancy and allows excellent differential diagnosis of a focal liver mass. CEUS regularly resolves an indeterminate result on CT/MRI and is exceptional as a problem-solving tool in liver imaging. CEUS has many similarities to contrast-enhanced CT/MRI but also unique and valuable differences, most showing the additional benefit of inclusion of CEUS with CT and MR for liver imaging.

\section{DECLARATIONS}

\section{Authors' contributions}

Made substantial contributions to conception and design of the study and date collection: Wilson SR, Merrill C

\section{Availability of data and materials}

Not applicable. 


\section{Financial support and sponsorship}

None.

\section{Conflicts of interest}

Wilson SR reports equipment support from Siemens, Samsung and Philips. Wilson SR reports a research grant from Samsung. Merrill C declared that there are no conflicts of interest.

\section{Ethical approval and consent to participate}

Not applicable.

\section{Consent for publication}

Not applicable.

\section{Copyright}

(C) The Author(s) 2020.

\section{REFERENCES}

1. Romanini L, Passamonti M, Aiani L, Cabassa P, Raieli G, et al. Economic assessment of contrast-enhanced ultrasonography for evaluation of focal liver lesions: a multicentre Italian experience. Eur Radiol 2007;17 Suppl 6:F99-106.

2. Claudon M, Dietrich CF, Choi BI, Cosgrove DO, Kudo M, et al. Guidelines and good clinical practice recommendations for contrast enhanced ultrasound (CEUS) in the liver--update 2012: a WFUMB-EFSUMB initiative in cooperation with representatives of AFSUMB, AIUM, ASUM, FLAUS and ICUS. Ultraschall Med 2013;34:11-29.

3. Claudon M, Dietrich CF, Choi BI, Cosgrove DO, Kudo M, et al; World Federation for Ultrasound in Medicine. European Federation of Societies for Ultrasound. Guidelines and good clinical practice recommendations for Contrast Enhanced Ultrasound (CEUS) in the liver - update 2012: a WFUMB-EFSUMB initiative in cooperation with representatives of AFSUMB, AIUM, ASUM, FLAUS and ICUS. Ultrasound Med Biol 2013;39:187-210.

4. Piscaglia F, Nolsøe C, Dietrich CF, Cosgrove DO, Gilja OH, et al. The EFSUMB Guidelines and Recommendations on the Clinical Practice of Contrast Enhanced Ultrasound (CEUS): update 2011 on non-hepatic applications. Ultraschall Med 2012;33:33-59.

5. Piscaglia F, Bolondi L; Italian Society for Ultrasound in Medicine and Biology (SIUMB) Study Group on Ultrasound Contrast Agents. The safety of Sonovue in abdominal applications: retrospective analysis of 23188 investigations. Ultrasound Med Biol 2006;32:1369-75.

6. Darge K, Papadopoulou F, Ntoulia A, Bulas DI, Coley BD, et al. Safety of contrast-enhanced ultrasound in children for non-cardiac applications: a review by the Society for Pediatric Radiology (SPR) and the International Contrast Ultrasound Society (ICUS). Pediatr Radiol 2013;43:1063-73.

7. Schwarze V, Marschner C, Negrão de Figueiredo G, Rübenthaler J, Clevert DA. Single-center study: evaluating the diagnostic performance and safety of contrast-enhanced ultrasound (CEUS) in pregnant women to assess hepatic lesions. Ultraschall Med 2020;41:29-35.

8. Averkiou M. Nonlinear pulsing schemes for the detection of ultrasound contrast agents. Available from: https://www.academia. edu/25447720/Nonlinear_pulsing_schemes_for_the_detection_of_ultrasound_contrast_agents. [Last accessed on 13 Aug 2020].

9. Burns PN, Wilson SR, Simpson DH. Pulse inversion imaging of liver blood flow: improved method for characterizing focal masses with microbubble contrast. Invest Radiol 2000;35:58-71.

10. Burns PN, Wilson SR. Focal liver masses: enhancement patterns on contrast-enhanced images--concordance of US scans with CT scans and MR images. Radiology 2007;242:162-74.

11. Wilson SR, Kim TK, Jang HJ, Burns PN. Enhancement patterns of focal liver masses: discordance between contrast-enhanced sonography and contrast-enhanced CT and MRI. AJR Am J Roentgenol 2007;189:W7-12.

12. Wilson SR, Burns PN. An algorithm for the diagnosis of focal liver masses using microbubble contrast-enhanced pulse-inversion sonography. AJR Am J Roentgenol 2006;186:1401-12.

13. Lyshchik A, Kono Y, Dietrich CF, Jang HJ, Kim TK, et al. Contrast-enhanced ultrasound of the liver: technical and lexicon recommendations from the ACR CEUS LI-RADS working group. Abdom Radiol (NY) 2018;43:861-79.

14. Burns PN, Wilson SR. Microbubble contrast for radiological imaging: 1. Principles. Ultrasound Q 2006;22:15-8.

15. Strobel D, Seitz K, Blank W, Schuler A, Dietrich C, et al. Contrast-enhanced ultrasound for the characterization of focal liver lesions-diagnostic accuracy in clinical practice (DEGUM multicenter trial). Ultraschall Med 2008;29:499-505.

16. Friedrich-Rust M, Klopffleisch T, Nierhoff J, Herrmann E, Vermehren J, et al. Contrast-Enhanced Ultrasound for the differentiation of benign and malignant focal liver lesions: a meta-analysis. Liver Int 2013;33:739-55.

17. Bhayana D, Kim TK, Jang HJ, Burns PN, Wilson SR. Hypervascular liver masses on contrast-enhanced ultrasound: the importance of washout. AJR Am J Roentgenol 2010;194:977-83.

18. Sporea I, Martie A, Bota S, Sirli R, Popescu A, et al. Characterization of focal liver lesions using contrast enhanced ultrasound as a first 
line method: a large monocentric experience. J Gastrointest Liver Dis 2014;23:57-63.

19. Kong WT, Wang WP, Huang BJ, Ding H, Mao F. Value of wash-in and wash-out time in the diagnosis between hepatocellular carcinoma and other hepatic nodules with similar vascular pattern on contrast-enhanced ultrasound. J Gastroenterol Hepatol 2014;29:576-80.

20. Murphy-Lavallee J, Jang HJ, Kim TK, Burns PN, Wilson SR. Are metastases really hypovascular in the arterial phase? The perspective based on contrast-enhanced ultrasonography. J Ultrasound Med 2007;26:1545-56.

21. Albrecht T, Blomley MJ, Burns PN, Wilson S, Harvey CJ, et al. Improved detection of hepatic metastases with pulse-inversion US during the liver-specific phase of SHU 508A: multicenter study. Radiology 2003;227:361-70.

22. Weskott HP. Detection and characterization of liver metastases. Radiologe 2011;51:469-74.

23. Laumonier H, Cailliez H, Balabaud C, Possenti L, Zucman-Rossi J, et al. Role of contrast-enhanced sonography in differentiation of subtypes of hepatocellular adenoma: correlation with MRI findings. AJR Am J Roentgenol 2012;199:341-8.

24. Kim TK, Jang HJ, Burns PN, Murphy-Lavallee J, Wilson SR. Focal nodular hyperplasia and hepatic adenoma: differentiation with lowmechanical-index contrast-enhanced sonography. AJR Am J Roentgenol 2008;190:58-66.

25. Dietrich CF, Mertens JC, Braden B, Schuessler G, Ott M, et al. Contrast-enhanced ultrasound of histologically proven liver hemangiomas. Hepatology 2007;45:1139-45.

26. Durot I, Wilson SR, Willmann JK. Contrast-enhanced ultrasound of malignant liver lesions. Abdom Radiol (NY) 2018;43:819-47.

27. Lee HS, Kim MJ, An C. How to utilize LR-M features of the LI-RADS to improve the diagnosis of combined hepatocellularcholangiocarcinoma on gadoxetate-enhanced MRI? Eur Radiol 2019;29:2408-16.

28. Fowler KJ, Potretzke TA, Hope TA, Costa EA, Wilson SR. LI-RADS M (LR-M): definite or probable malignancy, not specific for hepatocellular carcinoma. Abdom Radiol (NY) 2018;43:149-57.

29. Trillaud H, Bruel JM, Valette PJ, Vilgrain V, Schmutz G, et al. Characterization of focal liver lesions with SonoVue-enhanced sonography: international multicenter-study in comparison to CT and MRI. World J Gastroenterol 2009;15:3748-56.

30. Seitz K, Bernatik T, Strobel D, Blank W, Friedrich-Rust M, et al. Contrast-enhanced ultrasound (CEUS) for the characterization of focal liver lesions in clinical practice (DEGUM Multicenter Trial): CEUS vs. MRI--a prospective comparison in 269 patients. Ultraschall Med 2010;31:492-9.

31. Guang Y, Xie L, Ding H, Cai A, Huang Y. Diagnosis value of focal liver lesions with SonoVue ${ }^{\circledR}$-enhanced ultrasound compared with contrast-enhanced computed tomography and contrast-enhanced MRI: a meta-analysis. J Cancer Res Clin Oncol 2011;137:1595-605. 